\title{
Efeito da diluição do Rematitan Plus líquido sobre as dimensões de uma incrustação MOD
}

\section{Effects of the dilution of the Rematitan Plus liquid on the dimensions of an inlay MOD}

\author{
Renato Fabricio de Andrade WALDEMARIN* \\ Stella CROSARA* \\ Osvaldo ZANIQUELLI** \\ Osvaldo Luiz BEZZON***
}

\begin{abstract}
WALDEMARIN, R. F. A.; CROSARA, S.; ZANIQUELLI, O.; BEZZON, O. L. Efeito da diluição do Rematitan Plus líquido sobre as dimensões de uma incrustação MOD. Pesqui Odontol Bras, v. 14, n. 3, p. 283-286, jul./set. 2000.

Avaliou-se o efeito da diluição do Rematitan Plus líquido sobre as dimensões de uma incrustação MOD. A partir de uma matriz, foram confeccionados doze padrões de cera divididos em três grupos. Cada grupo foi incluído em uma diluição de Rematitan Plus líquido dentre aquelas recomendadas pelo fabricante (mínima, máxima e uma intermediária). A partir de marcas de referência feitas na matriz, as peças foram medidas no sentido M-D e V-L. Observou-se que a variação na diluição não alterava significativamente as dimensões da incrustação na maioria dos casos. Além disso, observou-se que as propriedades da cera utilizada como padrão para a confecção das incrustações foi mais significativa na alteração dimensional que a diluição do líquido do revestimento.
\end{abstract}

UNITERMOS: Adaptação marginal; Moldagem de cera para incrustações; Titânio.

\section{INTRODUÇÃO}

O uso de titânio em próteses vem ganhando espaço nos últimos anos devido a diversos fatores. WANG; FERTON ${ }^{7}$ (1996) em uma revisão da literatura citam: alta resistência à corrosão; baixo custo; baixa densidade; microdureza favorável e alta ductibilidade. Os estudos têm abordado o efeito de diversos tipos de revestimento e das formas e posições dos condutos de alimentação sobre a adaptação das peças de titânio; outros pesquisaram a adaptação das ligas de Ti-6Al-4V comparadas às de titânio puro e/ou compararam a adaptação de Ti puro com ligas de Ni-Cr e de Au-Pt-Pd usando peças que simulam coroas totais ou próteses parciais fixas.

De um modo geral, pode-se dizer que esses trabalhos concluíram que uma maior permeabilidade dos gases durante a fundição favorece a adaptação do Ti. Ainda, as comparações indicam a não existência de diferenças na adaptação entre peças de Ti puro e de Ni-Cr ou Au-Pt-Pd e diferenças entre Ti puro e Ti-6Al-4V.
Estudando a adaptação de peças de Au-Pd em PPF de 3, 4 e 5 elementos, SCHIFFLEGER et al. ${ }^{3}$ (1985) acharam que há um aumento nas dimensões das peças no sentido línguo-vestibular e uma diminuição nas dimensões no sentido mésio-distal, todas as medidas em relação à matriz usada.

O objetivo deste trabalho foi estudar o efeito de diversas diluições de Rematitan Plus líquido (líquido para espatulação do revestimento para fundições em titânio) sobre as dimensões de um padrão MOD e compará-los com os possiveis efeitos causados pelas alterações sofridas pelo padrão de cera durante a presa do revestimento.

\section{MATERIAIS E MÉTODOS}

Foi confeccionada uma matriz de aço em formato cilindrico, a qual foi usinada de forma a simular um preparo para uma incrustação MOD. Foram feitas marcas de referência sobre esta matriz, que permitiram a obtenção de medidas no sentido M-D e V-L. Tais medidas foram obtidas sob ampliação de 45 vezes usando-se um perfilômetro Nikon com

* Pós-Graduandos da Área de Reabilitação Oral; ** Professor Doutor da Disciplina de Materiais Dentários; *** Professor Titular da Disciplina de Clínica Integrada - Faculdade de Odontologia de Ribeirão Preto da USP. 
WALDEMARIN, R. F. A.; CROSARA, S.; ZANIQUELLI, O.; BEZZON, O. L. Efeito da diluição do Rematitan Plus líquido sobre as dimensões de uma incrustação MOD. Pesqui Odontol Bras, v. 14, n. 3, p. 283-286, jul./set. 2000.

precisão de um micrômetro. A forma e as dimensões da matriz estão mostradas na Figura 1.

Foram confeccionados sobre esta matriz doze padrões de cera, divididos em três grupos de quatro padrões. Cada grupo foi disposto em $90^{\circ}$ sobre condutos de alimentação conforme a Figura 2 e incluído em revestimento Rematitan Plus (pó/líquido) de acordo com a descrição abaixo.

- Grupo 1: Líquido composto de 100\% de Rematitan Plus líquido $(40 \mathrm{ml}$ ) para $250 \mathrm{~g}$ de Rematitan Plus pó.

- Grupo 2: Líquido composto de 80\% de Rematitan Plus líquido (32 ml) e $20 \%$ de água $(8 \mathrm{ml})$ para 250 g de Rematitan Plus pó.

- Grupo 3: Líquido composto de 60\% de Rematitan Plus líquido (24 $\mathrm{ml}$ ) e $40 \%$ de água $(16 \mathrm{ml})$ para 250 g de Rematitan Plus pó.

Após a presa do revestimento o mesmo passou,

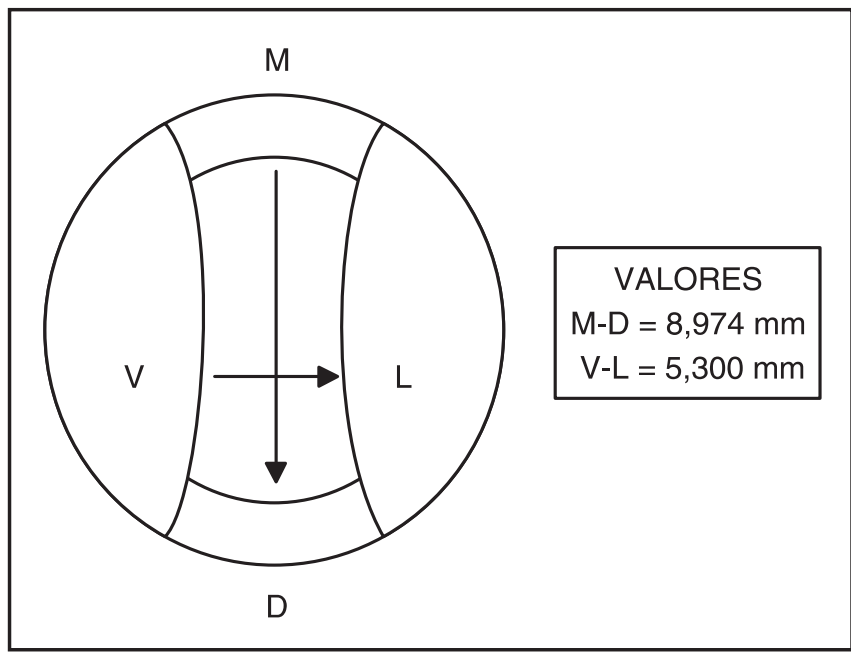

FIGURA 1 - Vista oclusal da matriz com a localização das marcas de referência. Os valores assinalados representam a média das medidas feitas.

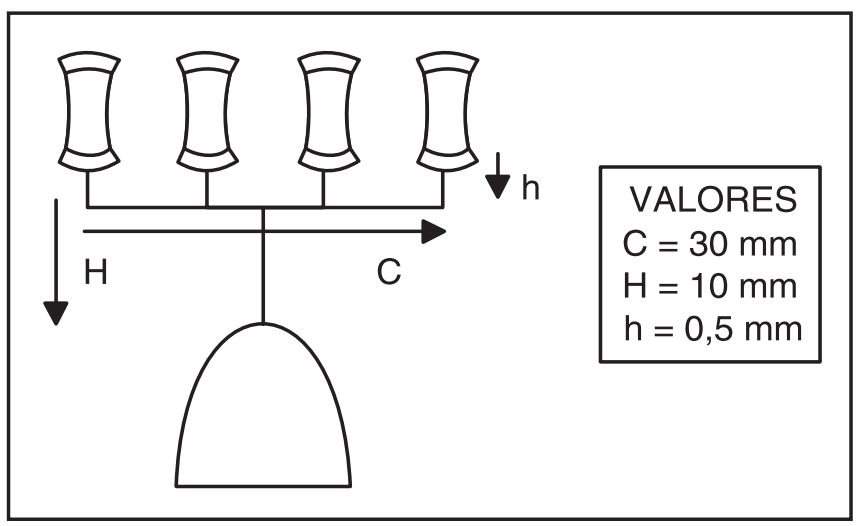

FIGURA 2 - Disposição dos padrões de cera sobre os condutos de alimentação. para remoção da cera, pela ciclagem térmica recomendada pelo fabricante e foi levado à uma máquina de fundição modelo Dentaurum/Rematitan. Tal máquina era composta de duas câmaras: a. uma câmara superior, onde um lingote de $22 \mathrm{~g}$ de titânio tipo 1 marca Rematitan era posicionado sobre um cadinho de cobre; tal câmara era pressurizada em atmosfera de argônio e o lingote era fundido por arco elétrico através de um eletrodo de tungstênio;

b. uma câmara inferior onde o molde era acomodado para receber a liga fundida, a qual era injetada sob pressão.

Depois de fundidas, as peças foram separadas de seus condutos, jateadas e reposicionadas sobre a matriz.

Com base nas marcas de referência feitas na matriz, foram obtidas cinco medidas por incrustação na direção M-D e cinco medidas por incrustação na direção V-L, totalizando vinte medidas por grupo em cada direção. Tais medidas foram obtidas sob ampliação de 45 vezes usando-se um perfilômetro Nikon com precisão de um micrômetro.

\section{RESULTADOS}

As médias e o desvio-padrão dos três grupos e da matriz em ambas direções medidas estão mostrados na Tabela 1 .

A análise de variância mostrou não haver diferença significativa na direção V-L entre os grupos, mas mostrou haver diferença a nivel menor que $1 \%$ quando estes eram comparados à matriz.

Na direção M-D a análise de variância associada ao teste de Tukey mostrou haver, entre o grupo $3(60 \%)$ e o grupo $1(100 \%)$, diferença significativa ao nível de $3 \%$. As diferenças entre os grupos 1 $(100 \%)$ e $2(80 \%)$ e a matriz foram significativas a nivel menor que $1 \%$; enquanto entre o grupo 3 $(60 \%)$ e a matriz a diferença foi significativa a nivel de $3 \%$.

TABELA 1 - Valores de média e desvio-padrão nos sentidos M-D e V-L para as incrustações e para a matriz.

\begin{tabular}{l|c|c|c|c}
\hline \hline \multirow{2}{*}{ Variável } & \multicolumn{2}{|c|}{ Medidas no sentido M-D } & \multicolumn{2}{c}{ Medidas no sentido V-L } \\
\cline { 2 - 5 } & $\begin{array}{c}\text { Média } \\
(\mathrm{mm})\end{array}$ & $\begin{array}{c}\text { Desvio- } \\
\text { padrão }\end{array}$ & $\begin{array}{c}\text { Média } \\
(\mathrm{mm})\end{array}$ & $\begin{array}{c}\text { Desvio- } \\
\text { padrão }\end{array}$ \\
\hline Grupo 1 & 9,137 & 0,080 & 5,203 & 0,029 \\
\hline Grupo 2 & 9,095 & 0,045 & 5,209 & 0,017 \\
\hline Grupo 3 & 9,072 & 0,093 & 5,211 & 0,013 \\
\hline Matriz & 8,974 & 0,004 & 5,300 & 0,012 \\
\hline \hline
\end{tabular}


WALDEMARIN, R. F. A.; CROSARA, S.; ZANIQUELLI, O.; BEZZON, O. L. Efeito da diluição do Rematitan Plus líquido sobre as dimensões de uma incrustação MOD. Pesqui Odontol Bras, v. 14, n. 3, p. 283-286, jul./set. 2000.

\section{DISCUSSÃO}

Dentre as diluições recomendadas pelo fabricante escolhemos: sem diluição (100\% de Rematitan Plus líquido); uma intermediária entre os extremos $180 \%$ de Rematitan Plus líquido e $20 \%$ de água) e a máxima recomendada pelo fabricante (60\% de Rematitan Plus líquido e 40\% de água).

As grandes diferenças estatísticas entre todos os grupos e o grupo matriz em ambos os casos indicam que, para este tipo de geometria, uma medida mais econômica pode ser adotada sem perda de qualidade no que tange à adaptação da peça. Ainda sobre este item vale observar que o grupo que mais se aproximou do grupo matriz foi o que teve maior diluição de seu componente líquido.

Segundo o fabricante, o revestimento expande menos à medida que se aumenta o conteúdo de água na diluição. Tal comportamento foi efetivamente observado nas medidas no sentido M-D, onde o valor da medida diminuiu à medida que se acrescentou água ao Rematitan Plus líquido.

Por outro lado, as medidas no sentido V-L da peça entraram em contradição com a afirmação do fabricante, pois aumentaram à medida que se acrescentou água ao Rematitan Plus líquido. Este fenômeno possivelmente pode ser explicado por meio do coeficiente de Poison, segundo o qual todo e qualquer corpo sólido, quando submetido a uma expansão (provocada por uma força de tração) em uma determinada direção, sofrerá uma contração em uma direção perpendicular àquela em que ele expandiu. A relação entre a contração e a expansão sofrida é fixa e própria de cada material. Dessa forma, quando o padrão de cera foi incluído no revestimento, este sofreu uma força de tração no sentido M-D devido ao contato do revestimento (que expandia) contra as paredes axiais de suas caixas proximais. Tal força de tração provocou um alongamento do padrão de cera nessa direção
(M-D) e uma contração na direção perpendicular a esta (V-L) devido ao coeficiente de Poison da cera.

Sendo assim, mesmo não se tendo pesquisado a cera, é de se esperar que valores menores de expansão do revestimento tenham provocado forças menores contra as paredes axiais do modelo. Isto provocou menor expansão deste no sentido M-D e, seguindo o raciocínio citado no parágrafo anterior, menor contração em seu sentido V-L. Menores contrações no sentido V-L possivelmente fizeram com que os valores obtidos das medidas nessa direção tenham se mostrado maiores à medida que se acrescentava água ao Rematitan Plus líquido.

\section{CONCLUSÕES}

Do acima exposto é possivel concluir que:

a. nenhuma diluição do Rematitan Plus líquido apresentou peças que se adaptassem perfeitamente à matriz sem usinagem;

b. a diluição de $60 \%$ de Rematitan Plus líquido e $40 \%$ de água é a que aparentemente necessitou de menos usinagem para adaptar-se;

c. o aumento no valor da diluição (dentro dos limites recomendados pelo fabricante) não alterou significativamente a adaptação das peças; exceção feita aos grupos 1 e 3 no sentido M-D;

d. a geometria particular das peças tipo incrustação MOD fez com que, em algumas partes destas, as alterações dimensionais não se comportassem conforme o esperado com base na análise exclusiva do comportamento do revestimento e, em outras, confirmam a menor expansão do revestimento à medida que se acrescenta água ao seu componente líquido;

e. a geometria da peça e as propriedades da cera utilizada como padrão para a confecção das incrustações possivelmente são mais significativas na alteração dimensional que a diluição do Rematitan Plus líquido.

WALDEMARIN, R. F. A.; CROSARA, S.; ZANIQUELLI, O.; BEZZON, O. L. Effects of the dilution of the Rematitan Plus liquid on the dimensions of an inlay MOD. Pesqui Odontol Bras, v. 14, n. 3, p. 283-286, jul./set. 2000.

The effects of the dilution of the Rematitan Plus liquid on the dimensions of an inlay MOD model were evaluated in this study. Twelve wax templates were built in a single matrix. Then they were separated into three groups. Each group was embedded in three different dilutions of Rematitan Plus, all of them recommended by the manufacturer (minimal, maximum and intermediate). From referential marks made on the matrix, the specimens were measured in M-D and B-L directions. We noticed that, in most cases, variations on the dilution did not change the dimensions of the inlays in a significant way. Furthermore, we observed that the properties of the wax used as a template for the construction of the inlays were more significant in the dimensional alterations than the dilution of the Rematitan Plus liquid.

UNITERMS: Marginal adaptation; Inlay casting wax; Titanium. 
WALDEMARIN, R. F. A.; CROSARA, S.; ZANIQUELLI, O.; BEZZON, O. L. Efeito da diluição do Rematitan Plus líquido sobre as dimensões de uma incrustação MOD. Pesqui Odontol Bras, v. 14, n. 3, p. 283-286, jul./set. 2000.

\section{REFERÊNCIAS BIBLIOGRÁFICAS}

1. BESIMO, C.; JEGER, C.; GUGGEnHEIM, R. Marginal adaptation of titanium frameworks produced by CAD/CAM techniques. Int J Prosthodont, v. 10, n. 6, p. 541-546, Nov./Dec. 1997.

2. CHAN, D. C. N.; BLACKMAN, R.; KAISER, D. A.; CHUNG, $\mathrm{K}$. The effect of sprue design on the marginal accuracy of titanium castings. J Oral Rehabil, v. 25, n. 6, p. 424-429, June 1998.

3. SCHIFFLEGER, B. E.; ZIEBERT, G. J.; DHURU, V. B.; BRANTLEY, W. A.; SIGAROUDI, K. Comparison of accuracy of multiunit one-piece castings. J Prosthet Dent, v. 54, n. 6, p. 770-776, Dec. 1985.

4. SYVERUD, M.; HERØ, H. Mold filling of Ti castings using investments with different gas permeability. Dent Mater, v. 11, n. 1, p. 14-18, Jan. 1995.
5. SYVERUD, M.; OKABE, T.; HERØ, H. Casting of Ti-6Al-4V alloy compared with pure $\mathrm{Ti}$ in an Ar-arc casting machine. Eur J Oral Sci, v. 103, n. 5, p. 327-330, Oct. 1995.

6. VALDERRAMA, S.; ROEKEL, N. V.; ANDERSSON, M.; GOODACRE, C. J.; MUNOZ, C. A. A comparison of the marginal and internal adaptation of titanium and gold-platinum-palladium metal ceramic crowns. Int $\mathbf{J}$ Prosthodont, v. 8, n. 1, p. 29-36, Jan./Feb. 1995.

7. WANG, R. R.; FENTON, A. Titanium for prosthodontic applications: a review of the literature. Quintessence Int, v. 27, n. 6, p. 401-408, June 1996.

8. WATANABE, I.; WATKINS, J. H.; NAKAJIMA, H.; ATSUTA, M.; OKABE, T. Effect of pressure difference on the quality of titanium casting. J Dent Res, v. 76, n. 3, p. 773-779, Mar. 1997.

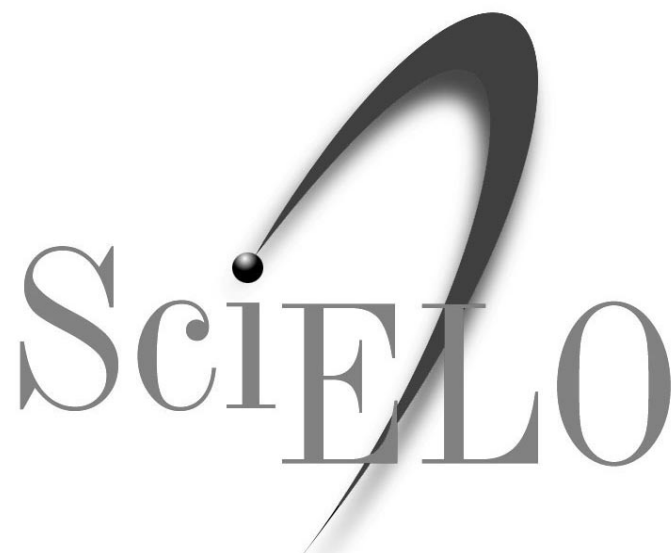

\section{Scientific Electronic Library Online http://www.scielo.br Parceria: FAPESP/BIREME}

SciELO - Scientific Electronic Library Online é uma coleção eletrônica de revistas científicas disponíveis na Internet. A coleção traz os textos completos de artigos científicos, bases de dados e relatórios de uso e de impacto da literatura científica publicada no site.

Em operação desde 1997, atualmente estão disponíveis 29 revistas brasileiras nas áreas de ciências biológicas e da saúde, ciências sociais e humanas, ciências agrárias, física etc. Novas publicações são incluídas regularmente em várias especialidades.

Entre as revistas de nossa coleção, está a Pesquisa Odontológica Brasileira.

$$
\begin{aligned}
& \text { Consulte a Pesquisa Odontológica Brasileira (Revista de Odontologia da } \\
& \text { USP) na SciELO - http:/ / www.scielo.br/rousp }
\end{aligned}
$$

